\title{
Algorithms and Performance of Small Baseline Acoustic Sensor Arrays
}

\author{
Brian M. Sadler ${ }^{a}$, Richard J. Kozick ${ }^{b}$, Sandra L. Collier $^{a}$ \\ ${ }^{a}$ Army Research Laboratory, Adelphi, MD \\ ${ }^{b}$ Bucknell University, Lewisburg, PA
}

\begin{abstract}
Aeroacoustic sensing is well motivated due to its passive nature and low bandwidth, and processing with array baselines of one to a few meters is well studied and useful. However, arrays of this size present difficulties in manufacture and deployment. Motivated by the desire to develop smaller, cheaper (perhaps "disposable") sensor packages, we consider performance for arrays with small baselines. The performance of angle estimation operating roughly in the $[30,500] \mathrm{Hz}$ regime is limited by the observed signal-to-noise ratio (SNR) and propagation in the turbulent atmosphere. Scattering results in a reduction of spatial coherence, which places fundamental limits on angle estimation accuracy. Physics-based statistical models for the scattering have enabled prediction of network performance, including detection, angle estimation, time-delay estimation, and geolocation. In the present work, we focus on angle estimation accuracy for the short baseline case. While a large aperture is desirable to improve angle estimation, the propagation produces a rolloff in the spatial coherence that ultimately degrades the performance despite increasing the array aperture. We characterize this tradeoff analytically via Cramér-Rao bounds, as a function of SNR, frequency, range, sensor array geometry, and propagation conditions. The results clearly favor shorter ranges and higher frequencies when employing small array baselines.
\end{abstract}

Keywords: Unattended ground sensors, acoustics, array processing, angle of arrival estimation

\section{INTRODUCTION}

Aeroacoustics are useful for passive surveillance due to the mature sensing technology available. The low data bandwidth allows for sophisticated signal processing in a sensor network, including detection, geolocation, classification, and tracking. In this paper we consider array processing with relatively small baselines. This is motivated by the desire to keep the overall sensor node small, covert, and easily deployable. For example, some experimental results with a 5-inch 5-element array and an interferometric algorithm have been reported. ${ }^{1}$ However, the exploitation of small baselines is fundamentally limited by propagation through the turbulent atmosphere, and the long wavelengths encountered. So, it is of interest to study the impact of propagation and other factors on small-baseline acoustic array processing performance.

There are several competing factors to consider with respect to the size of the array baseline. Large baselines are desired for angle of arrival (AOA) accuracy. In this paper we focus on the frequency range $f \in[30,500] \mathrm{Hz}$. This results in wavelengths $\lambda$ of about $2 / 3$ to 11 meters, and short baselines may be a relatively small fraction of a wavelength.

Scattering from atmospheric turbulence results in random fluctuations in the phase and amplitude of propagating wavefronts, and results in a spatial coherence loss that grows with spatial separation. ${ }^{2,3}$ This coherence loss strongly affects achievable AOA accuracy as the sensor separations grow, and this effect becomes more significant at higher frequencies and longer ranges. ${ }^{4}$

In addition to scattering from atmospheric turbulence, there are several causes of attenuation of sound waves propagating in air. ${ }^{5,6}$ These include spherical spreading, absorption, refraction, ground reflections, and other factors. Although sources of interest, such as vehicles and aircraft, may be loud, these factors combine to produce significant transmission loss, especially at higher frequencies. The source spectrum may be predominantly in the lower frequencies. Spherical spreading produces a roughly $6 \mathrm{~dB}$ loss per doubling of distance. Above 500 $\mathrm{Hz}$, absorption causes at least $2 \mathrm{~dB}$ of loss per $\mathrm{km}$, and increases rapidly with frequency. When sensors are deployed above the ground, the direct and reflected waves cause spectral nulls due to mutual cancelation, which 
impacts elevation angle estimation. Ground waves are essentially low passed filtered, depending on range. And, diffraction around obstacles, as well as refraction due to temperature and wind gradients, creates shadow zones. With regard to attenuation, in this paper, the SNR used throughout is that observed at the sensor.

From the above discussion, we see that there are competing factors with respect to the array baseline size. Unlike the ideal case of plane wave propagation, arbitrarily extending the baseline in the case of aeroacoustics does not lead to better and better AOA estimation performance. To study the tradeoffs, and to provide AOA performance bounds, we employ Cramér-Rao bounds (CRBs) that incorporate turbulence effects. ${ }^{7,8}$ The analysis includes propagation conditions, SNR, sensor array geometry, source range and frequency, and spatial coherence. The AOA estimation bounds characterize the loss beyond simple plain wave assumptions (which may be quite optimistic), and so provide tighter lower bounds. We also include simulation results that address the achievability of the bounds with practical algorithms.

The rest of the paper is outlined as follows. To study small baselines, we first consider a two-sensor array, developing the observation model, CRBs, and AOA estimators. We then generalize to a ring of sensors.

\section{MODEL}

Consider a single, narrowband, far-field source that is observed at a two-sensor array at normal incidence after propagation through a random, inhomogeneous medium. We model the complex envelope of the sensor signals as a complex, Gaussian random vector,

$$
\widetilde{\mathbf{z}} \sim \mathrm{CN}\left(e^{j \chi} \sqrt{(1-\Omega) S} \mathbf{a},(\Omega S) \mathbf{\Gamma} \circ\left(\mathbf{a a}^{H}\right)+\sigma^{2} \mathbf{I}\right) .
$$

In our notation, o denotes element-wise product, $(\cdot)^{T}$ and $(\cdot)^{H}$ denote transpose and Hermitian transpose, respectively, and $\mathbf{I}$ is the identity matrix. The average power of the source at each sensor is $S$, the average power of the noise at each sensor is $\sigma^{2}$, the signal to noise ratio (SNR) at each sensor is

$$
\mathrm{SNR}=\frac{S}{\sigma^{2}}
$$

Setting the phase at the first sensor to be zero, we define the steering vector as follows,

$$
\mathbf{a}=\left[\begin{array}{c}
1 \\
e^{j \phi}
\end{array}\right]
$$

The phase angle, $\phi$, is related to the source angle of arrival (AOA), $\theta$, as

$$
\begin{aligned}
\phi & =\frac{2 \pi}{\lambda} \rho \sin \theta=\frac{\omega}{c_{0}} \rho \sin \theta \\
\theta & =\arcsin \left(\frac{\lambda}{2 \pi \rho} \phi\right)=\arcsin \left(\frac{c_{0}}{\omega \rho} \phi\right),
\end{aligned}
$$

where $\rho$ is the spacing between the sensors, $\lambda$ is the wavelength, $\omega$ is the radian frequency, and $c_{0}$ is the speed of sound. The turbulent propagation medium is characterized by the saturation, $\Omega$, and the spatial coherence, $\gamma$, where the matrix $\boldsymbol{\Gamma}$ is

$$
\boldsymbol{\Gamma}=\left[\begin{array}{ll}
1 & \gamma \\
\gamma & 1
\end{array}\right]
$$

The saturation $\Omega \in[0,1]$, where $\Omega \approx 0$ signifies weak scattering and $\Omega \approx 1$ signifies strong scattering. The spatial coherence is real-valued, with $0 \leq \gamma \leq 1$. The signal phase $\chi$ in (1) depends on the source phase and the source range, $d_{0}$.

For AOAs in the range $-\pi / 2<\theta<\pi / 2$, it follows from (4) that the phase $-2 \pi \rho / \lambda<\phi<2 \pi \rho / \lambda$, so the sensor spacing must satisfy $\rho<\lambda / 2$ to guarantee AOA identifiability. For frequencies in the range $f \in[30,500]$ $\mathrm{Hz}$, the wavelength varies from $\lambda=c_{0} / f=11.1 \mathrm{~m}$ to $0.67 \mathrm{~m} \approx 26.3$ inches, based on the speed of sound $c_{0}=334$ $\mathrm{m} / \mathrm{s}$. Therefore sensor spacings of $\rho \leq 12$ in are smaller than a half-wavelength for frequencies up to $500 \mathrm{~Hz}$. 
The turbulence parameters, $\Omega$ and $\gamma$, depend on the source frequency $\omega$, source range $d_{0}$, sensor spacing $\rho$, and the weather conditions. The following model is valid for source frequencies $(\omega /(2 \pi))$ in the range from about 30 to $500 \mathrm{~Hz}$ and for sensor separations $(\rho)$ on the order of a few meters or less. ${ }^{7}$ The saturation is given by

$$
\Omega=1-\exp \left(-2 \mu d_{0}\right),
$$

where $\mu$ is the extinction coefficient of the first moment, and

$$
\begin{aligned}
\mu(\omega) & \approx\left\{\begin{array}{ll}
4.03 \times 10^{-7}\left(\frac{\omega}{2 \pi}\right)^{2}, & \text { mostly sunny } \\
1.42 \times 10^{-7}\left(\frac{\omega}{2 \pi}\right)^{2}, & \text { mostly cloudy }
\end{array}, \frac{\omega}{2 \pi} \in[30,500] \mathrm{Hz}\right.
\end{aligned}
$$

The factor $\kappa_{1}$ (weather) depends on the weather conditions as in (8). The spatial coherence $\gamma$ is determined by $\nu$, the extinction coefficient of the second moment, according to

$$
\gamma=\frac{\exp \left(-\nu d_{0}\right)-(1-\Omega)}{\Omega}
$$

The saturation parameter $\Omega$ models the effect of scattering from atmospheric turbulence. A fully saturated signal, $\Omega=1$, corresponds to strong scattering and a weakly saturated signal, $\Omega \approx 0$, corresponds to weak scattering. The spatial coherence models both scattering (through its dependence on $\Omega$ ) and diffraction (through its dependence on $\nu$ ). to

The extinction coefficient depends on the weather conditions, source frequency, and sensor spacing according

$$
\nu=\kappa_{2}\left(\text { weather) } \omega^{2} \rho^{5 / 3}\right.
$$

where the factor $\kappa_{2}$ (weather) is given by

$$
\kappa_{2}(\text { weather })=\frac{0.137}{c_{0}^{2}}\left(\frac{C_{T}^{2}}{T_{0}^{2}}+\frac{22}{3} \frac{C_{v}^{2}}{c_{0}^{2}}\right)
$$

and where $C_{T}^{2}$ and $C_{v}^{2}$ are the structure-function parameters for temperature and velocity fluctuations, respectively, and $T_{0}$ is a representative value of the mean temperature in Kelvin. The first and second terms in (12) represent the contributions of temperature and wind fluctuations to the coherence, respectively. Table 1, which is reproduced from Kozick et al., ${ }^{7}$ provides estimated values for these terms under different weather conditions based on turbulence models. ${ }^{2,3}$ Table 1 also includes estimated values for $\mu$ that are consistent with the model in (8). The equation for $\nu$ in (11) is valid only for sensor separations $\rho \ll \mathcal{L}_{\text {eff }}$, where eddy size $\mathcal{L}_{\text {eff }}$ is tabulated in the rightmost column of Table 1. For larger values of $\rho$, the extinction coefficient $\nu$ peaks at $2 \mu$, so $\gamma$ in (10) is limited to the range $[0,1]$.

We can combine the preceding expressions as follows to show the dependence of $\Omega$ and $\gamma$ on the source frequency $\omega$, source range $d_{0}$, sensor spacing $\rho$, and the weather conditions:

$$
\begin{aligned}
& \Omega=1-\exp \left(-2 \kappa_{1} \text { (weather) } \omega^{2} d_{0}\right) \\
& \gamma=\frac{\exp \left(-\kappa_{2}(\text { weather }) \omega^{2} \rho^{5 / 3} d_{0}\right)-\exp \left(-2 \kappa_{1}(\text { weather }) \omega^{2} d_{0}\right)}{1-\exp \left(-2 \kappa_{1}(\text { weather }) \omega^{2} d_{0}\right)} .
\end{aligned}
$$

These expressions show that saturation $\Omega \rightarrow 1$ and spatial coherence $\gamma \rightarrow 0$ as the source frequency and range increase, as the sensor spacing increases, and as the intensity of the turbulent fluctuations increase such as is characteristic of sunny and windy weather conditions. These detrimental factors act to limit AOA estimation performance, as we describe next. 


\begin{tabular}{|l|r|r|c|r|r|}
\hline Atmospheric condition & $\begin{array}{c}\mu^{-1}(\mathrm{~m}) \\
\text { at } 50 \mathrm{~Hz}\end{array}$ & $\begin{array}{c}\mu^{-1}(\mathrm{~m}) \\
\text { at } 200 \mathrm{~Hz}\end{array}$ & $\begin{array}{c}C_{T}^{2} / T_{0}^{2} \\
\left(\mathrm{~m}^{-2 / 3}\right)\end{array}$ & $\begin{array}{c}(22 / 3) C_{v}^{2} / c_{0}^{2} \\
\left(\mathrm{~m}^{-2 / 3}\right)\end{array}$ & $\begin{array}{c}\mathcal{L}_{\text {eff }} \\
(\mathrm{m})\end{array}$ \\
\hline \hline Mostly sunny, light wind & 990 & 62 & $2.0 \times 10^{-5}$ & $8.0 \times 10^{-6}$ & 100 \\
\hline Mostly sunny, moderate wind & 980 & 61 & $7.6 \times 10^{-6}$ & $2.8 \times 10^{-5}$ & 91 \\
\hline Mostly sunny, strong wind & 950 & 59 & $2.4 \times 10^{-6}$ & $1.3 \times 10^{-4}$ & 55 \\
\hline Mostly cloudy, light wind & 2900 & 180 & $1.5 \times 10^{-6}$ & $4.4 \times 10^{-6}$ & 110 \\
\hline Mostly cloudy, moderate wind & 2800 & 180 & $4.5 \times 10^{-7}$ & $2.4 \times 10^{-5}$ & 75 \\
\hline Mostly cloudy, strong wind & 2600 & 160 & $1.1 \times 10^{-7}$ & $1.2 \times 10^{-4}$ & 28 \\
\hline
\end{tabular}

Table 1. Modeled turbulence quantities and inverse extinction coefficients for various atmospheric conditions. The atmospheric conditions are described quantitatively in Ostashev and Wilson. ${ }^{2}$ The second and third columns give the inverse extinction coefficients at $50 \mathrm{~Hz}$ and $200 \mathrm{~Hz}$, respectively. These values indicate the distance at which random fluctuations in the complex signal become strong. The fourth and fifth columns represent the relative contributions of temperature and wind fluctuations to the field coherence. The sixth column is the effective integral length scale for the scattered sound field; at sensor separations greater than this value, the coherence is "saturated."

\section{CRAMÉR-RAO BOUND AND A TWO-ELEMENT ARRAY}

Next we derive the Cramér-Rao bound (CRB) on the phase $\phi$ and AOA $\theta$ in (1). In particular, to gain insight into the impact of the various factors, in this section we focus on the two-element array case. We use results from Collier et al. ${ }^{8}$ (see eqn (53) in that reference for the 2-element CRB case). In the following sections we consider other array geometries with more sensors. We derive the CRB on $\phi$ assuming that all other parameters in (1) are known. The CRB on the phase is obtained from the Fisher information (FI), $J_{\phi \phi}$, as

$$
\operatorname{CRB}(\hat{\phi})=\frac{1}{J_{\phi \phi}}
$$

The CRB on the AOA, $\theta=g(\phi)=\arcsin \left(\phi c_{0} /(\omega \rho)\right)$ in (5), is obtained from the formula for transformation of parameters, ${ }^{9}$

$$
\operatorname{CRB}(\hat{\theta})=\left(\frac{\partial g}{\partial \phi}\right)^{2} \operatorname{CRB}(\hat{\phi})=\frac{\operatorname{CRB}(\hat{\phi})}{\frac{\rho^{2} \omega^{2}}{c_{0}^{2}}\left[1-\left(\frac{c_{0}}{\rho \omega} \phi\right)^{2}\right]} .
$$

As is well-known, the CRB on the AOA in (16) gets smaller with increasing sensor spacing and frequency. Equivalently, since $\rho \omega / c_{0}=2 \pi \rho / \lambda$, the CRB is reduced as the sensor spacing as a fraction of the wavelength is increased.

The FI can be expressed as

$$
J_{\phi \phi}=2 \cdot \operatorname{SNR} \frac{1-\Omega}{1+\Omega \cdot \operatorname{SNR}\left[2+\Omega \cdot \operatorname{SNR}\left(1-\gamma^{2}\right)\right]}+2 \cdot \operatorname{SNR} \cdot \Omega \frac{1-\Omega\left(1-\gamma^{2}\right)}{\Omega\left[2+\Omega \cdot \operatorname{SNR}\left(1-\gamma^{2}\right)\right]+\frac{1}{\operatorname{SNR}}} .
$$

The first term in (17) vanishes when $\Omega=1$ (strong scattering) and the second term vanishes when $\Omega=0$ (no scattering). The bound on the variance of any unbiased AOA estimator is obtained from (13), (14), (17), and (16).

Now we consider the Fisher information on the phase estimation for some special cases, which are all approximations obtained from (17).

\section{No scattering: $\Omega=0$}

$$
J_{\phi \phi}=2 \cdot \mathrm{SNR}
$$

The FI in (18) with no scattering is the familiar result for a deterministic signal, where the factor of 2 corresponds to the coherent combining of the signals at the two sensors. The coherence, $\gamma$, is not a relevant parameter when $\Omega=0$. 
2. Full saturation: $\Omega=1$, coherence $0 \leq \gamma^{2}<1$

$$
\begin{array}{rlrl}
J_{\phi \phi} & = & 2 \frac{\gamma^{2}}{\left(1-\gamma^{2}\right)+\frac{2}{\mathrm{SNR}}+\frac{1}{\mathrm{SNR}^{2}}} & \\
& \rightarrow & & \text { for SNR } \gg 1 \text { and } \gamma^{2}<1-2 / \mathrm{SNR} \\
& \rightarrow \quad & \text { for SNR } \gg 1 \text { and } \gamma^{2}>1-2 / \mathrm{SNR} .
\end{array}
$$

With full saturation, (20) shows that the performance bound is determined by the coherence when the SNR is large. If the coherence is very close to 1, then (21) shows that the performance is SNR-limited. However, comparing (21) with the case of no scattering in (18), the fluctuations caused by the turbulence effectively reduce the SNR by $3 \mathrm{~dB}$, even when the coherence is nearly perfect.

3. High SNR: SNR $\gg 1,0<\Omega \leq 1$, and $0 \leq \gamma^{2}<1$

$$
\begin{aligned}
J_{\phi \phi} & =\frac{2(1-\Omega)}{2 \Omega+\Omega^{2} \cdot \operatorname{SNR}\left(1-\gamma^{2}\right)}+\frac{2\left[1-\Omega\left(1-\gamma^{2}\right)\right]}{\Omega\left(1-\gamma^{2}\right)} \\
& \rightarrow 2 \frac{\frac{1}{\Omega}-1+\gamma^{2}}{1-\gamma^{2}} \quad \text { for SNR } \gg 1 \text { and } \gamma^{2}<1-2 / \mathrm{SNR} .
\end{aligned}
$$

This case corresponds to high SNR with turbulent scattering, since the coherence is imperfect, $0 \leq \gamma^{2}<1$, and the saturation is in the range $0<\Omega \leq 1$. The FI in (22) is limited by the coherence. The first term in (22) is inversely proportional to SNR, so the FI approaches (23) at high SNR, which generalizes (20) to the case with $0<\Omega<1$.

4. Perfect coherence: $\gamma^{2}=1$

$$
\begin{aligned}
& J_{\phi \phi}=\quad 2 \cdot \mathrm{SNR} \frac{\Omega \cdot \mathrm{SNR}+(1-\Omega)}{2 \Omega \cdot \mathrm{SNR}+1} \\
& \rightarrow \quad 2 \cdot \mathrm{SNR} \quad \text { if } \Omega=0 \text { (no scattering) } \\
& \rightarrow \quad \operatorname{SNR} \frac{1}{1+\frac{1}{2 \cdot \operatorname{SNR}}} \quad \text { if } \Omega=1 \\
& \rightarrow \quad \text { SNR } \quad \text { if SNR } \gg 1 \text { and } \Omega>0 .
\end{aligned}
$$

The FIs in (25) and (27) show that with high SNR and no coherence losses, scattering $(\Omega>0)$ effectively reduces the SNR by $3 \mathrm{~dB}$ relative to the case of no scattering.

5. Zero coherence: $\gamma^{2}=0$

$$
\begin{array}{rlrl}
J_{\phi \phi} & = & \\
& \rightarrow & & \\
& & 2\left(\frac{1}{\Omega}-1\right) & \text { if SNR } \gg 1 \text { and } \Omega>1 / \mathrm{SNR}
\end{array}
$$

With zero coherence, (29) shows that the FI is saturation-limited at high SNR. Note that $J_{\phi \phi}=0$ when $\Omega=1$ and $\gamma=0$ because the model (1) contains no information about the phase.

Note that the SNR-limited FI in (18) and (21) is achievable when there is no scattering or when the coherence is nearly perfect, i.e., $\gamma^{2} \gg 1-2 / \mathrm{SNR}$. Coherence losses lead to the coherence-limited FI in (23). 


\section{CRB EXAMPLES FOR A TWO-ELEMENT ARRAY}

Based on the results of the previous section, we consider the performance for the two-element array case. Figures 1 and 2 show the saturation $\Omega$ and coherence $\gamma$, plotting (13) and (14) for sensor separation $\rho=12$ in., source frequencies over 30 to $500 \mathrm{~Hz}$, source ranges from 10 to $10,000 \mathrm{~m}$, and different weather conditions. Note that, generally speaking, sunny and windy conditions produce more turbulence, whereas cloudy and calm are more benign. Figure 1 shows $\Omega$ for sunny and cloudy cases. For example, for sunny conditions, at source of $d_{0}=500 \mathrm{~m}$, full saturation is predicted to occur at frequencies above $100 \mathrm{~Hz}$. Figure 2 depicts spatial coherence between the two array elements, for the various parameter ranges, now with wind introduced. These plots indicate how the coherence rolls off with increasing range and frequency. Note that, although the coherence does decline with higher ranges and frequencies, it is predicted to remain relatively high for this small array aperture, even at ranges approaching $1 \mathrm{~km}$. Expanding the aperture size will result in further significant decrease in coherence.

Figure 3 shows the CRBs on the AOA for $\mathrm{SNR}=30 \mathrm{~dB}$, sensor separation $\rho=12 \mathrm{in}$., source frequencies in the range from 30 to $500 \mathrm{~Hz}$, source ranges from 10 to $10,000 \mathrm{~m}$, and two different weather conditions. We observe the significant upward shifting (worsening) of the performance as the range is increased. Comparing the left and right panels, we see that the change in propagation conditions has a dramatic impact on the achievable AOA performance. For source ranges that are $2 \mathrm{~km}$ and less, the CRBs on AOA decrease with increasing frequency, as expected, because the (fixed) baseline is proportionally larger as a function of wavelength. However, for longer source ranges of $5 \mathrm{~km}$ and $10 \mathrm{~km}$, the CRBs on AOA increase with frequency (for most weather conditions) because of the significant coherence losses occurring due to the atmospheric turbulence.

The dots and diamonds are included in Figure 3 to illustrate the SNR-limited and coherence-limited performance regimes. The lines with black dots are from eqn (27), which corresponds to perfect coherence and high SNR. Note that the CRBs for small range $(10 \mathrm{~m})$ are close to this approximation, but that significantly worse performance can be expected in many cases over that predicted without accounting for the turbulence. The diamonds correspond to the coherence-limited, high SNR approximation using (23).

Figure 4 contains plots of the CRB on the differential phase $\phi$ for the entire range of $\Omega$ and $\gamma$ variations, for two different SNR values. The CRB with no scattering, $\Omega=0$ in Figures 4(a) and (b), is inversely proportional to SNR as in (18). The case of perfect coherence, $\gamma=1$ in Figures $4(\mathrm{a})$ and (b), follows (25) for $\Omega=0$ and (27) for $\Omega>0$. Even with high SNR $=30 \mathrm{~dB}$, the accuracy achievable is sensitive to the coherence and saturation. We also note that, comparison with the coherence-limited, high SNR approximation (23) for $\Omega>0$ show good agreement with the $30 \mathrm{~dB}$ case; the CRB is coherence-limited whenever $\gamma^{2}$ differs from unity by more than about $1 / \mathrm{SNR}$.

\section{SIMULATION EXAMPLES FOR A TWO-ELEMENT ARRAY}

Based on the complex normal observation model in eqn (1), we simulate AOA estimation for two algorithms, and compare with the appropriate CRBs. From $(1), \widetilde{\mathbf{z}} \sim \mathrm{CN}(\mathbf{m}, \mathbf{C})$ with mean vector, covariance matrix, and probability density function, respectively,

$$
\begin{aligned}
\mathbf{m} & =e^{j \chi} \sqrt{(1-\Omega) S} \mathbf{a} \\
\mathbf{C} & =(\Omega S) \mathbf{\Gamma} \circ\left(\mathbf{a a}^{H}\right)+\sigma^{2} \mathbf{I} \\
f(\widetilde{\mathbf{z}}) & =\frac{1}{\pi^{2} \operatorname{det}(\mathbf{C})} \exp \left\{-\operatorname{trace}\left[\mathbf{C}^{-1}(\widetilde{\mathbf{z}}-\mathbf{m})(\widetilde{\mathbf{z}}-\mathbf{m})^{H}\right]\right\} .
\end{aligned}
$$



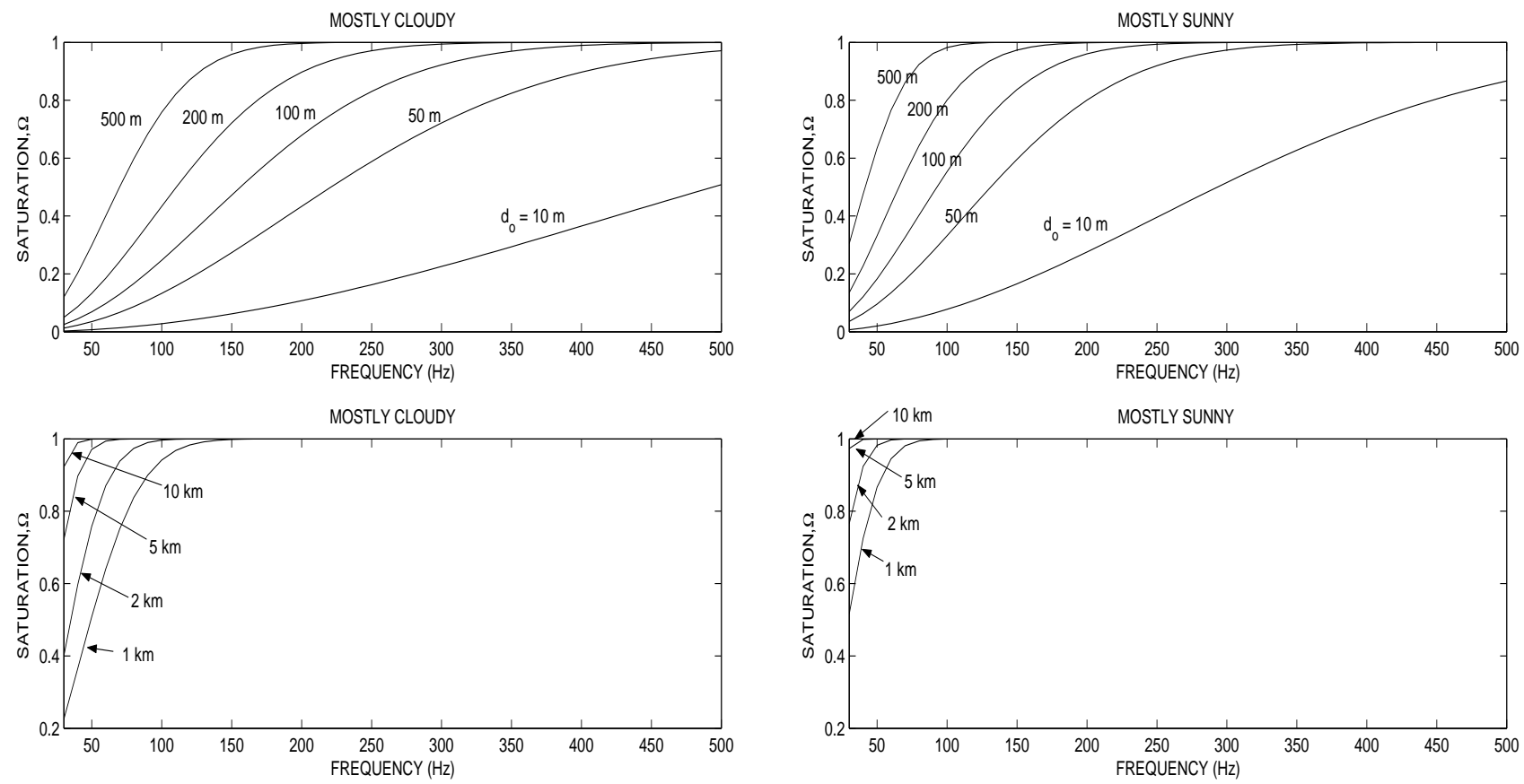

(a)

(b)

Figure 1. Saturation vs frequency, with sensor spacing $\rho=12$ inches $(0.3 \mathrm{~m})$, range $=(10,50,100,200,500) \mathrm{m}$ in upper panels and $(1,2,5,10) \mathrm{km}$ in lower panels, and (a) cloudy conditions, (b) sunny conditions.
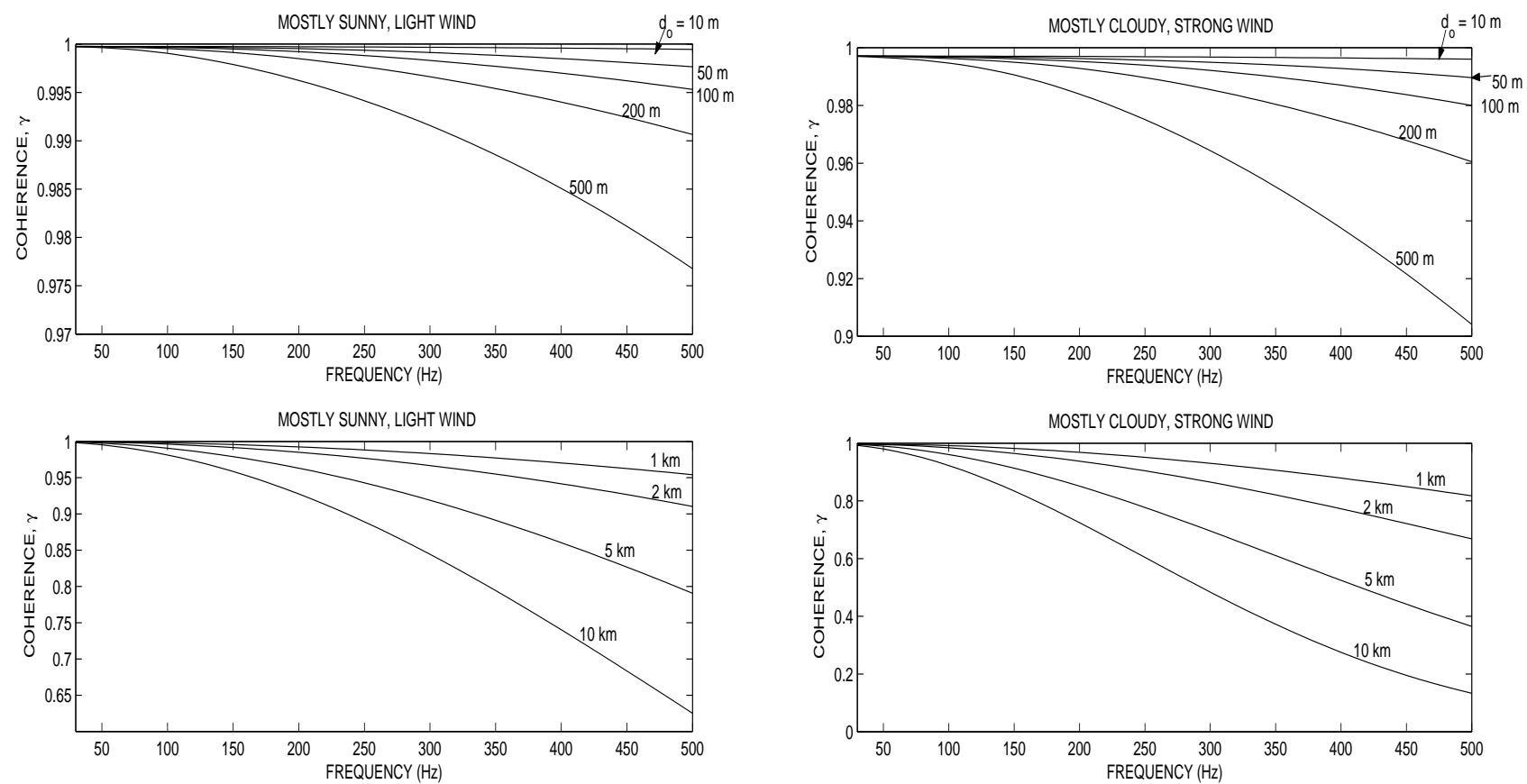

(a)

(b)

Figure 2. Spatial coherence vs frequency, with sensor spacing $\rho=12$ inches $(0.3 \mathrm{~m})$, range $=(10,50,100,200,500) \mathrm{m}$ in upper panels and $(1,2,5,10) \mathrm{km}$ in lower panels, and (a) sunny light wind, and (b) cloudy, strong wind conditions. 


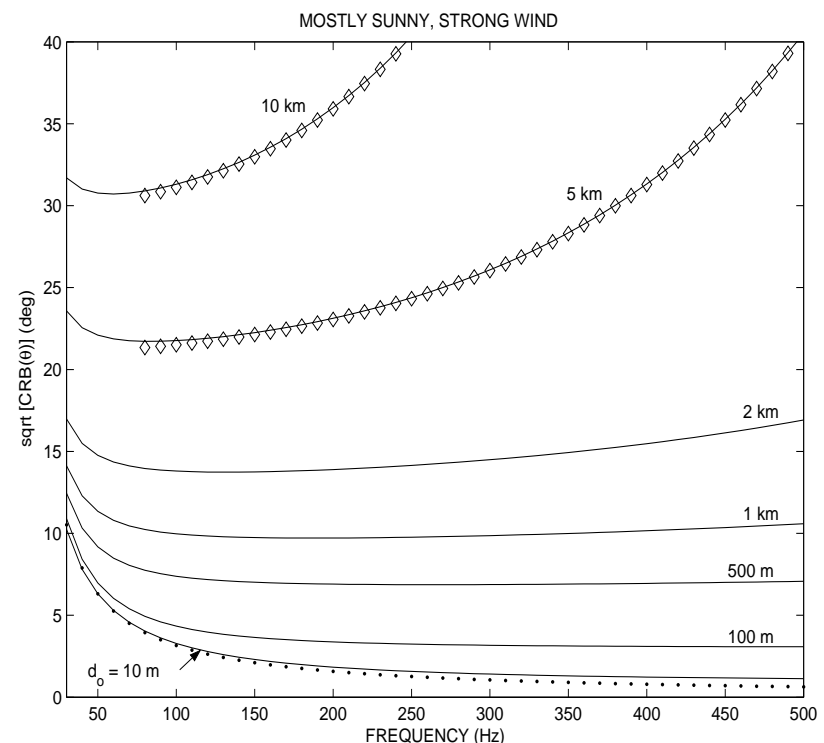

(a)

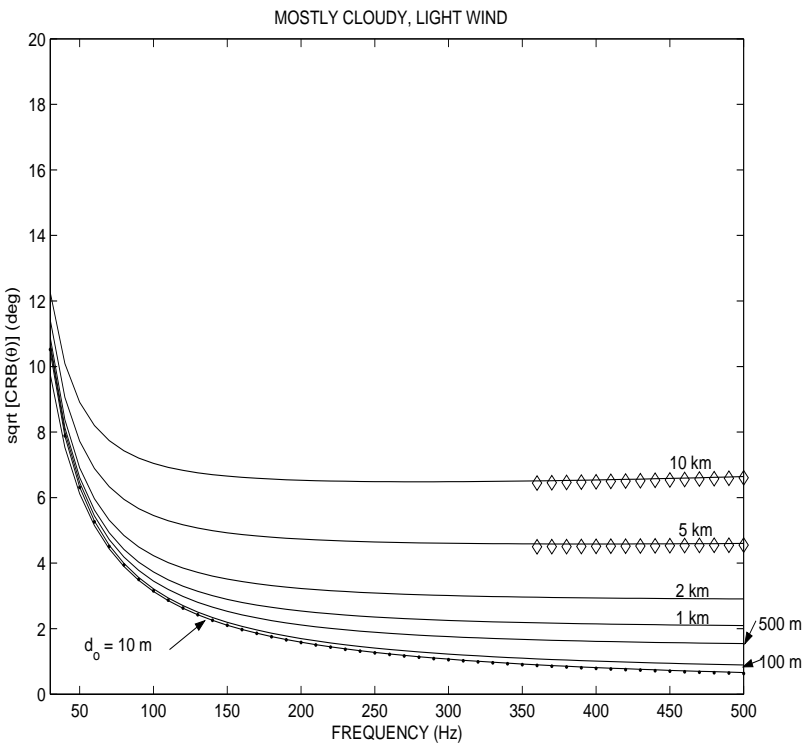

(b)

Figure 3. $\sqrt{\mathrm{CRB}(\hat{\theta})}=$ square root of $\mathrm{CRB}$ on $\mathrm{AOA}$ for $\mathrm{SNR}=30 \mathrm{~dB}$, sensor spacing $\rho=12$ inches $(0.3 \mathrm{~m})$, range $=$ $10,100,500,1,000,2,000,5,000$, and 10,000 $\mathrm{m}$, and two propagation conditions. The black dots are based on (27) with perfect coherence and high SNR, and the diamonds are based on (23) for high SNR (coherence-limited performance).

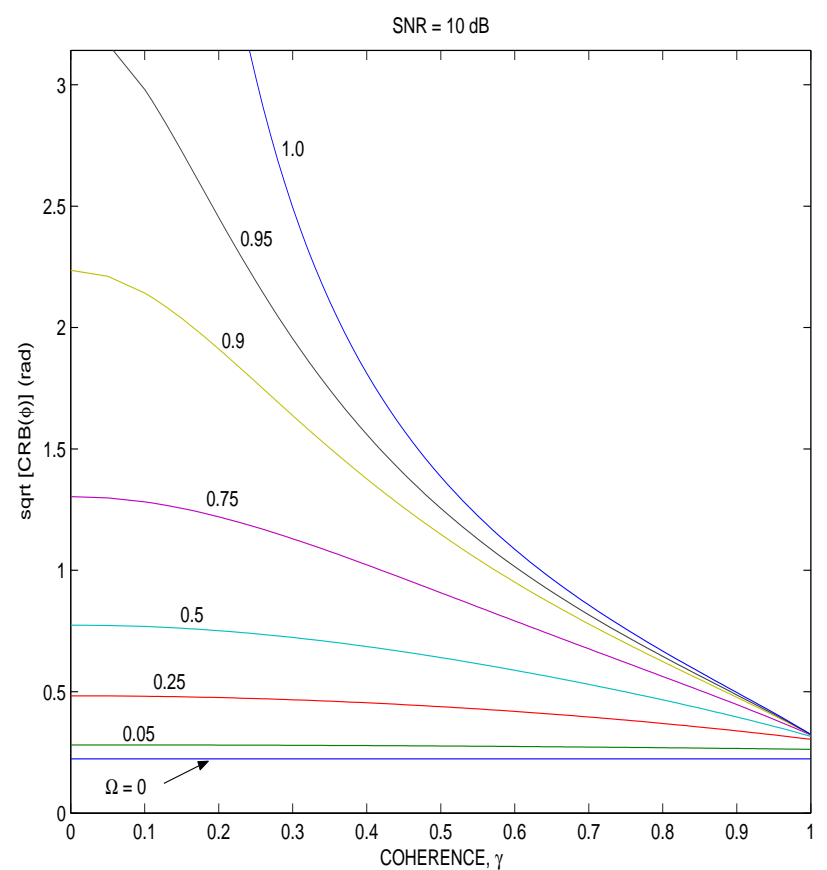

(a)

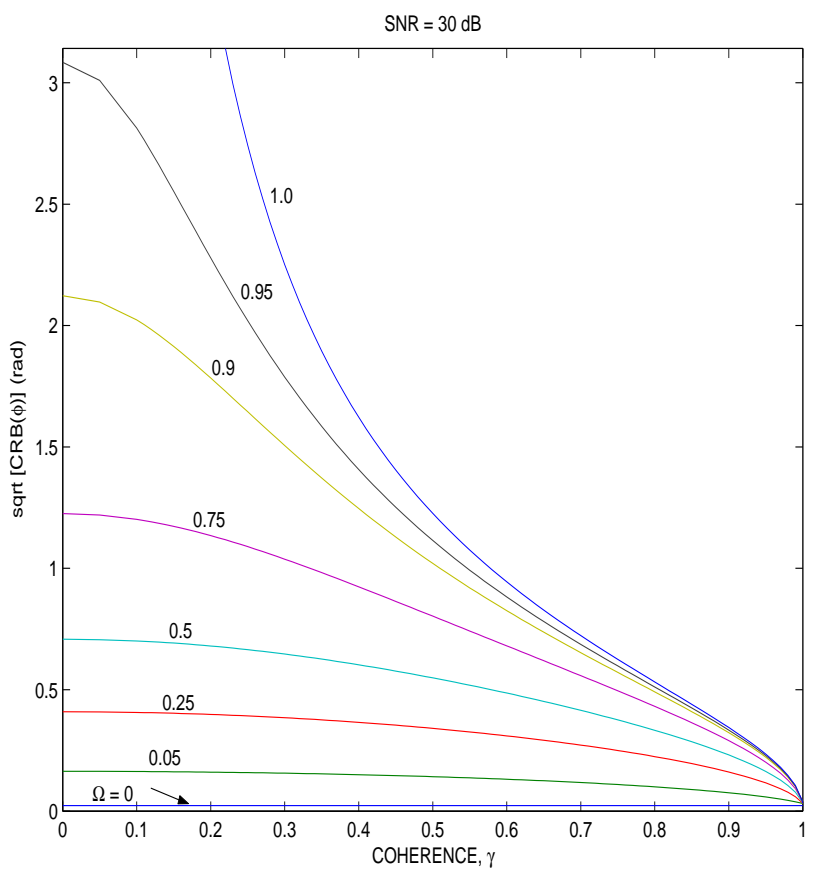

(b)

Figure 4. $\sqrt{\operatorname{CRB}(\hat{\phi})}=$ square root of $\mathrm{CRB}$ on phase in (15) and (17) versus saturation $\Omega$ and coherence $\gamma$ for (a) SNR $=10 \mathrm{~dB}$ and (b) SNR $=30 \mathrm{~dB}$ in. Results in (b) are very close to the high-SNR, coherence-limited approximation in (23) for $\Omega>0$. 
For computer simulation, we generate realizations of complex, Gaussian random vectors $\widetilde{\mathbf{z}}$ with mean and covariance in (1) with $\chi=0$, corresponding to a single snapshot, with

$$
\begin{array}{rlrl}
\widetilde{\mathbf{z}} & =(\sqrt{1-\Omega} \mathbf{1}+\sqrt{\Omega} \widetilde{\mathbf{v}}) \circ \mathbf{a}+\widetilde{\mathbf{w}} & \widetilde{\mathbf{w}} & \sim \mathrm{CN}\left(\mathbf{0}, \mathrm{SNR}^{-1} \mathbf{I}\right) \\
\widetilde{\mathbf{v}} & \sim \mathrm{CN}(\mathbf{0}, \boldsymbol{\Gamma}) & \mathbf{a} & =\left[\begin{array}{c}
1 \\
e^{j \phi}
\end{array}\right] \\
\mathbf{\Gamma} & =\left[\begin{array}{ll}
1 & \gamma \\
\gamma & 1
\end{array}\right]=\mathbf{G} \mathbf{G}^{T} & \mathbf{G} & =\frac{1}{\sqrt{2}}\left[\begin{array}{rr}
\sqrt{1+\gamma} & -\sqrt{1-\gamma} \\
\sqrt{1+\gamma} & \sqrt{1-\gamma}
\end{array}\right] \\
\widetilde{\mathbf{v}} & =\mathbf{G} \widetilde{\mathbf{u}} & & \widetilde{\mathbf{u}} \sim \mathrm{CN}(\mathbf{0}, \mathbf{I}), \text { independent from } \widetilde{\mathbf{w}} .
\end{array}
$$

The correlated scattered components in $\widetilde{\mathbf{v}}$ are generated using the square root matrix $\mathbf{G}$ with the iid random variables in $\widetilde{\mathbf{u}}$.

We consider two estimators for the phase parameter. With $\widetilde{\mathbf{z}}=\left[z_{1}, z_{2}\right]^{T}$ and $z_{i}=\left|z_{i}\right| \exp \left(j \angle z_{i}\right)$, the first estimator simply forms the phase difference $(\mathrm{PD})$ between the observations,

$$
\widehat{\phi}_{P D}=\angle\left(z_{2}\right)-\angle\left(z_{1}\right) \text {. }
$$

The PD estimator is independent of the propagation parameters $(\Omega$ and $\gamma)$, the absolute phase $(\chi)$, and the SNR.

Our second estimator is maximum likelihood (ML), which assumes that $\Omega, \gamma, \chi$, and SNR are known, and is given by

$$
\begin{aligned}
\widehat{\phi}_{M L} & =\underset{\phi}{\operatorname{argmax}} \ln f(\widetilde{\mathbf{z}}) \\
& =\underset{\phi}{\operatorname{argmax}}\left\{\Omega \gamma\left|z_{1}\right|\left|z_{2}\right| \cos \left(\phi+\angle z_{1}-\angle z_{2}\right)+\sqrt{1-\Omega}\left[\Omega(1-\gamma)+\mathrm{SNR}^{-1}\right]\left|z_{2}\right| \cos \left(\phi-\angle z_{2}+\chi\right)\right\} .
\end{aligned}
$$

The expression in (39) is obtained through algebraic manipulation of (30)-(32) in (38). The ML estimator may have limited usefulness in practice because it assumes $\Omega$ and $\gamma$ are known, but provides an optimal estimate for comparison with the simple PD estimator in (37). When the SNR is high and the ML estimation error is small, an approximate solution to (39) is

$$
\begin{aligned}
\widehat{\phi}_{M L} & \approx \frac{b_{0}\left(\angle z_{2}-\chi\right)+b_{1}\left(\angle z_{2}-\angle z_{1}\right)}{b_{0}+b_{1}} \\
\text { where } \quad b_{0} & =\sqrt{1-\Omega}\left[\Omega(1-\gamma)+\mathrm{SNR}^{-1}\right]\left|z_{2}\right|, \quad b_{1}=\Omega \gamma\left|z_{1}\right|\left|z_{2}\right| .
\end{aligned}
$$

In the case of no scattering $(\Omega=0)$, the ML estimate is $\widehat{\phi}_{M L}=\angle z_{2}-\chi$. In this case, $z_{1}$ is ignored since perfect knowledge of the absolute phase, $\chi$, is assumed. In the case of full saturation $(\Omega=1)$, the ML estimate is identical to the PD estimate in (37). For $0<\Omega<1$, the ratio of weight factors $b_{0}, b_{1}$ in (39)-(41) is, at high SNR,

$$
\frac{b_{1}}{b_{0}} \approx \frac{\gamma\left|z_{1}\right|}{\sqrt{1-\Omega}(1-\gamma)} .
$$

The PD and ML estimators are potentially most different when $b_{0} \gg b_{1}$, which occurs when the SNR is low or when the coherence is very low. An AOA estimate is obtained from the phase estimate using (5),

$$
\widehat{\theta}=\arcsin \left(\frac{c_{0}}{\omega \rho} \widehat{\phi}\right) .
$$

If the argument of the $\arcsin$ in $(43)$ is outside the range $[-1,1]$, then we truncate the value to -1 or +1 so that $\widehat{\theta}$ is in the range from $-90^{\circ}$ to $90^{\circ}$. 
Simulation examples are presented in Figures 5 and 6, where 30,000 runs are used to compute the root meansquared error (RMSE) for each data point. Generally, there is a gap between the estimators and the CRBs. Also notice that the simple PD estimator performed almost on a par with the optimal ML estimator. Figure 5 shows results for a 12-inch sensor spacing. In Figure 5(a), even at a short range of $25 \mathrm{~m}$, an SNR of $10 \mathrm{~dB}$ is insufficient to produce accurate AOA estimates except perhaps at the high edge of the frequency band.* In Figure 5(b), despite the longer range of $500 \mathrm{~m}$, better performance is obtained due to the high SNR of $30 \mathrm{~dB}$. In both cases, estimation accuracy is poor at the low end of the band due to insufficient array baseline.

Figure 6 shows results for a 3-inch sensor spacing, with both (a) and (b) at a high SNR of $40 \mathrm{~dB}$, and for two different ranges (50 and $100 \mathrm{~m}$ ). AOA estimator performance is reasonably accurate for such a small baseline, but it declines sharply below about $100 \mathrm{~Hz}$ and follows the CRB. Even with 30,000 trials, the estimates displayed significant variability for frequencies that correspond to intermediate values of $\Omega$ in the range from about 0.2 to less than 1.0. This is evident in Figure 6 where the estimator RMSE curves are not smooth over part of the frequency range.

\section{CIRCULAR ARRAY}

In this section we show angle estimation accuracy predictions via the CRB for AOA, for a six-element circular array. We adapt the general results in Collier et al. ${ }^{8}$ to this case. The SNR is fixed at $30 \mathrm{~dB}$, and the radius of the array is varied from 0.1 to 1 meter. Near normal incidence is assumed, with the array 1 meter above ground. Results are shown in Figure 7. Figure 7(a) is for cloudy and calm conditions (low turbulence), while figure 7(b) is for sunny and windy (high turbulence). Four source ranges are shown for each propagation condition. For this high SNR case, good AOA estimation accuracy is predicted down to the radius of $0.1 \mathrm{~m}$, even at the higher frequencies and longer ranges.

\section{CONCLUSIONS}

We have presented performance analysis for estimating angle of arrival of an acoustic source, employing relatively small array apertures. The results reveal the interplay of the frequency, SNR at the sensor, propagation conditions, and array size, and predict reasonable performance is achievable in many conditions with small apertures. Because the bounds incorporate the effects of atmospheric turbulence, they are considerably more accurate than an ideal plane wave assumption, and so should be useful for bounding system performance. We also remind the reader that the SNR used throughout the paper is taken at the sensor, so when comparing results with varying range but equal SNR, the longer ranges correspond to louder sources. SNR gain can be achieved by adding more sensors, for example, in a circular configuration.

Examining the coherence and saturation, we can identify performance regimes of interest. Large eddies, a characteristic of sunny conditions, have a stronger effect on the bounds than do the small eddies characteristic of high winds. Turbulence intensity is strongest under mostly sunny and windy conditions, and so array performance is predicted to be worst for this case. On the other hand, in calm and cloudy conditions, performance predictions are often close to that obtained with no turbulence.

\section{ACKNOWLEDGMENTS}

The authors thank D. K. Wilson and T. Pham for continuing collaboration and insight on this work.

${ }^{*}$ Note that at 30 and $40 \mathrm{~Hz}$ in Figure 5(a), the MSE on AOA estimation is less than the CRB. This is a result of the data truncation discussed after (43) so that the argument of the arcsin is in the range $[-1,1]$. 


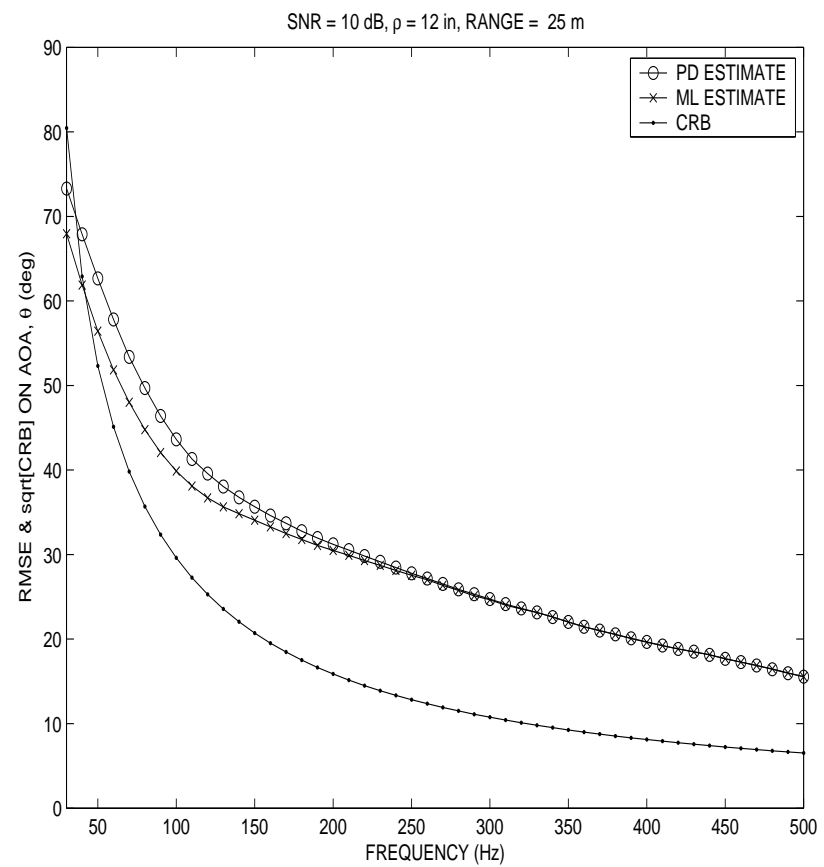

(a)

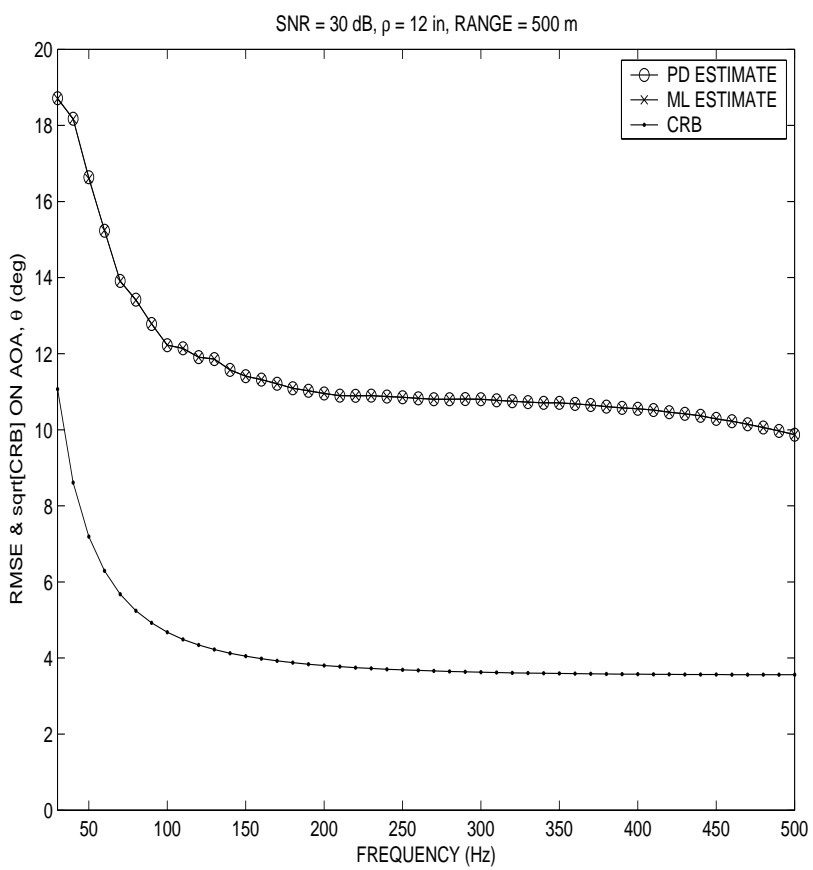

(b)

Figure 5. AOA simulation examples and CRBs with sensor separation $\rho=12$ in, and mostly sunny, moderate wind conditions. (a) $\mathrm{SNR}=10 \mathrm{~dB}$, range $=25 \mathrm{~m}$. (b) $\mathrm{SNR}=30 \mathrm{~dB}$, range $=500 \mathrm{~m}$.

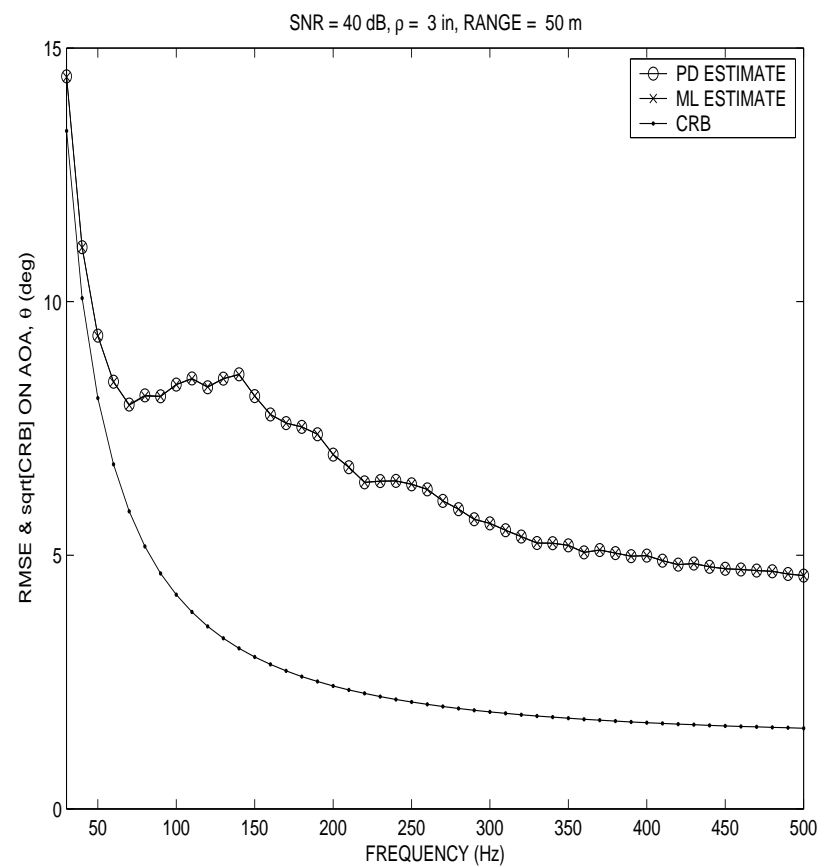

(a)

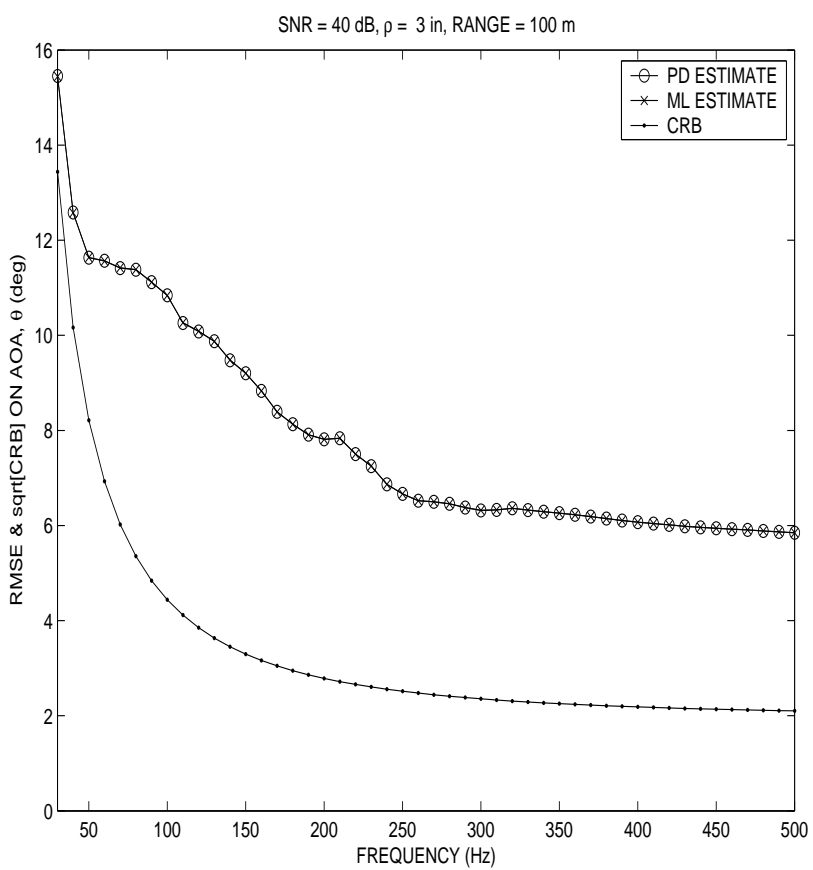

(b)

Figure 6. AOA simulation examples and CRBs with $\mathrm{SNR}=40 \mathrm{~dB}$, sensor separation $\rho=3$ in, and mostly sunny, moderate wind conditions. (a) Range $=50 \mathrm{~m}$, (b) range $=100 \mathrm{~m}$. 

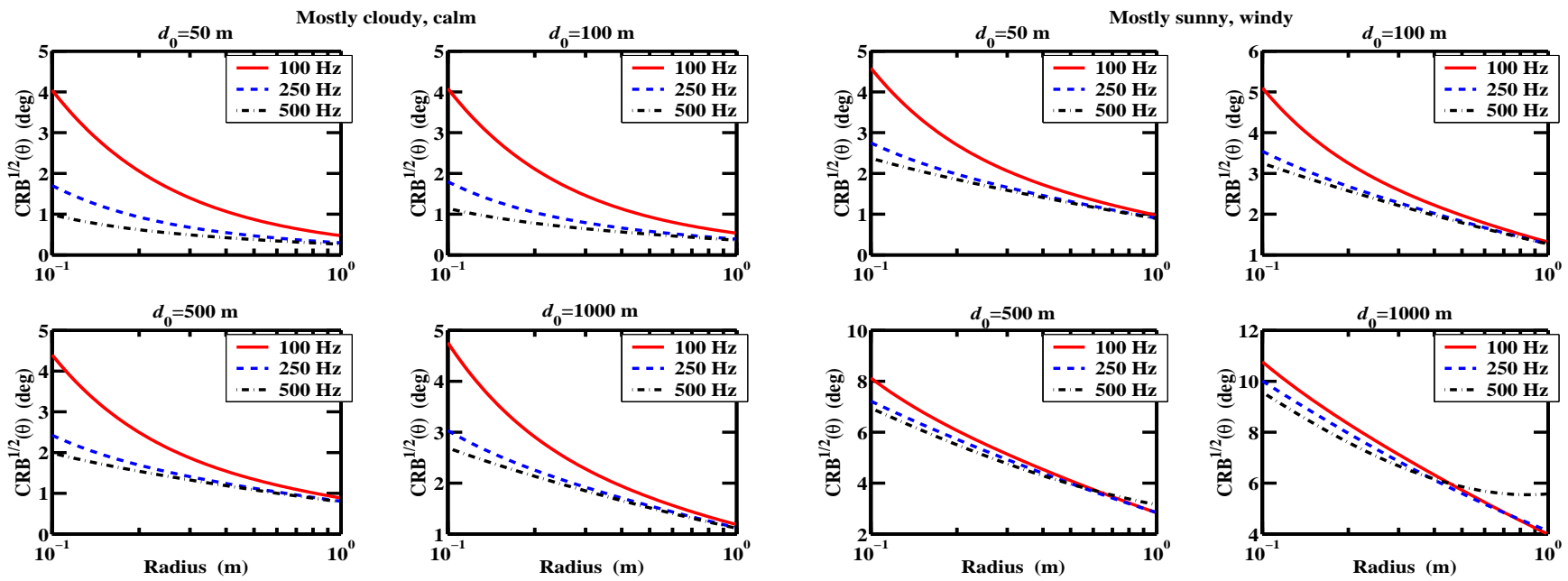

(a)

(b)

Figure 7. Sqrt of the $\mathrm{CRB}$ on $\mathrm{AOA}$ for a six-element circular array, whose radius varies from 0.1 to 1.0 meter, and $\mathrm{SNR}=30 \mathrm{~dB}$. Curves are parameterized by source frequency, with (a) cloudy calm and (b) sunny windy propagation conditions.

\section{REFERENCES}

1. G. Prado, G. Succi, et al., "A multi-mission unattended ground sensor," SPIE AeroSense 2002 Symposium, Orlando, FL, April 2002.

2. V. E. Ostashev and D. K. Wilson, "Relative contributions from temperature and wind velocity fluctuations to the statistical moments of a sound field in a turbulent atmosphere," Acta Acustica, vol. 86, pp. 260-268, 2000 .

3. D.K. Wilson, "A turbulence spectral model for sound propagation in the atmosphere that incorporates shear and buoyancy forcings", J. Acoust. Soc. Am., vol. 108, no. 5, Pt. 1, pp. 2021-2038, Nov. 2000.

4. R.J. Kozick, B.M. Sadler, "Source localization with distributed sensor arrays and partial spatial coherence," IEEE Transactions on Signal Processing, vol. 52, no. 3, pp. 601-616, March 2004.

5. I. Rudnick, "Propagation of sound in the open air," chapter 3 in Handbook of Noise Control (McGraw-Hill, 1957), C. M. Harris, ed.

6. T. F. W. Embleton, "Tutorial on sound propagation outdoors," J. Acoust. Soc. Am., vol. 100, no. 1, pp. 31-48, 1996.

7. R.J. Kozick, B.M. Sadler, D.K. Wilson, "Signal Processing and Propagation for Aeroacoustic Networks," to appear in Frontiers in Distributed Sensor Networks, S.S. Iyengar and R.R. Brooks (Eds.), (CRC Press, 2004).

8. S. L. Collier and D. K. Wilson, "Performance bounds for passive sensor arrays operating in a turbulent medium: Plane-wave analysis," J. Acoust. Soc. Am., vol. 113, no. 5, pp. 2704-2718, May 2003.

9. S. M. Kay, Fundamentals of Statistical Signal Processing, Estimation Theory (Prentice-Hall, 1993). 